\title{
Alterstice
}

Revue internationale de la recherche interculturelle

International Journal of Intercultural Research

Revista International de la Investigacion Intercultural

\section{Penser la recherche interculturelle : le défi des diversités}

\section{Anahy Gajardo et Yvan Leanza}

Volume 1, numéro 1, 2011

Penser la recherche interculturelle : le défi des diversités

URI : https://id.erudit.org/iderudit/1077586ar

DOI : https://doi.org/10.7202/1077586ar

Aller au sommaire du numéro

\section{Éditeur(s)}

Alterstice

ISSN

1923-919X (numérique)

Découvrir la revue

Citer ce document

Gajardo, A. \& Leanza, Y. (2011). Penser la recherche interculturelle : le défi des diversités. Alterstice, 1(1), 3-7. https://doi.org/10.7202/1077586ar

\section{Résumé de l'article}

Entré dans le vocabulaire courant des disciplines (psychologie, pédagogie, sociologie, etc.) et des champs de pratiques et de recherches (éducation, santé, social, management, etc.), le terme d' " interculturel » se caractérise par la multiplicité de ses champs d'application, l'instabilité de ses significations et un flou sémantique certain.

Qu'en est-il de la recherche dite « interculturelle » ? Que signifie ce terme dans le contexte du travail scientifique ? Peut-on identifier une conception partagée - ou paradigmatique - du terme lorsqu'il est mobilisé dans le champ scientifique ? Peut-on dégager des épistémologies, des méthodologies, ou pour le moins des pratiques et des caractéristiques scientifiques communes à ce champ de recherche?

Dans le cadre de ce premier numéro d'Alterstice, il nous est apparu indispensable de soulever ces questions. En effet, "faire de la recherche interculturelle » implique aussi de la penser, de s'interroger sur les conceptions, les usages, les épistémologies et les méthodologies que nous, chercheures et chercheurs, associons à ce terme qui qualifie nos pratiques scientifiques ou nos objets de recherche. 


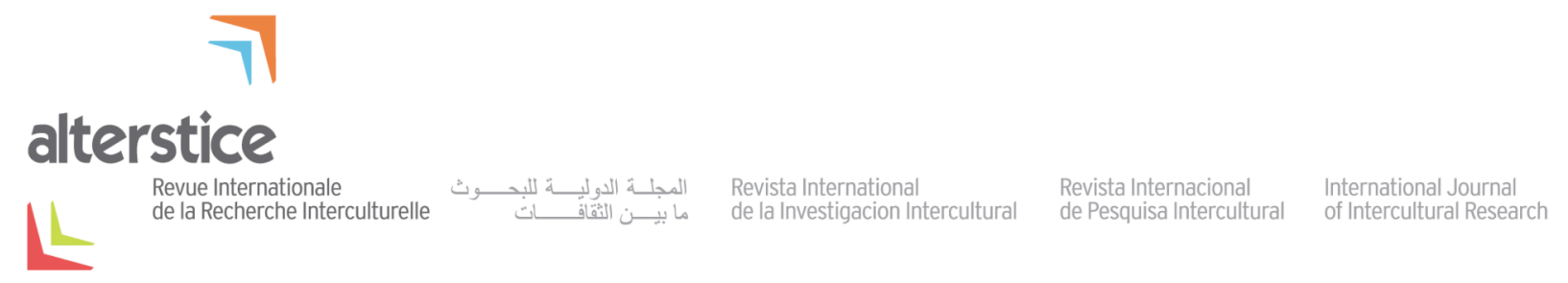

INTRODUCTION THÉMATIQUE

\section{Penser la recherche interculturelle : le défi des diversités}

Anahy Gajardo ${ }^{1}$ et Yvan Leanza ${ }^{2}$

\section{Résumé}

Entré dans le vocabulaire courant des disciplines (psychologie, pédagogie, sociologie, etc.) et des champs de pratiques et de recherches (éducation, santé, social, management, etc.), le terme d' "interculturel » se caractérise par la multiplicité de ses champs d'application, l'instabilité de ses significations et un flou sémantique certain.

Qu'en est-il de la recherche dite " interculturelle »? Que signifie ce terme dans le contexte du travail scientifique? Peut-on identifier une conception partagée - ou paradigmatique - du terme lorsqu'il est mobilisé dans le champ scientifique? Peut-on dégager des épistémologies, des méthodologies, ou pour le moins des pratiques et des caractéristiques scientifiques communes à ce champ de recherche?

Dans le cadre de ce premier numéro d'Alterstice, il nous est apparu indispensable de soulever ces questions. En effet, " faire de la recherche interculturelle » implique aussi de la penser, de s'interroger sur les conceptions, les usages, les épistémologies et les méthodologies que nous, chercheures et chercheurs, associons à ce terme qui qualifie nos pratiques scientifiques ou nos objets de recherche.

\section{Rattachement des auteurs \\ ${ }^{1}$ Université de Neuchâtel, Neuchâtel, Suisse'; ${ }^{2}$ Université Laval, Québec, Canada}

\section{Correspondance}

anahy.gajardo@unine.ch

\section{Mots clés}

interculturel

\section{Pour citer cet article :}

Gajardo, A et Leanza, Y. (2011). Penser la recherche interculturelle : le défi des diversités. Alterstice, 1(1), 3-8. 


\section{L'interculturel dans tous ses états}

Entré dans le vocabulaire courant des disciplines (psychologie, pédagogie, sociologie, etc.) et des champs de pratiques et de recherches (éducation, santé, social, management, etc.), le terme d' " interculturel » se caractérise par la multiplicité de ses champs d'application, l'instabilité de ses significations et un flou sémantique certain. Imprécis, vague et polysémique, il est néanmoins omniprésent dans le vocabulaire de nombreuses pratiques professionnelles, des sciences humaines et sociales, ainsi que dans les rhétoriques politiques. La plupart des formations ayant un rapport avec l'humain traitent, même de façon marginale, de cette question; rares sont les écoles supérieures et les universités qui n'offrent pas un ou plusieurs cours sur le thème. Cette notion apparaît aussi de plus en plus souvent dans les normes des professions: tout professionnel de l'humain (du médecin à l'enseignant, en passant par les éducateurs spécialisés, et bien d'autres) se doit d'être au moins sensibilisé au sujet, si ce n'est pleinement formé et posséder des compétences dites interculturelles. En tant que modalité de gestion de la diversité culturelle, le mot occupe aujourd'hui une place de choix dans le champ discursif politique; cela, au niveau des villes, des régions, des États ou même des organisations internationales, comme le Conseil de l'Europe et son livre blanc sur le dialogue interculturel (2008). L'interculturel apparaît ainsi comme " une notion caméléon, s'adaptant à divers champs d'application et véhiculant, sous le même terme, des conceptions et des pratiques non similaires, parfois non comparables ni compatibles » (Gajardo, Dervin et Lavanchy, 2011, p. 7).

Qu'en est-il de la recherche dite " interculturelle »? Que signifie ce terme dans le contexte du travail scientifique? Que qualifie-t-il? Hormis le fait de " prendre la culture au sérieux » (Dasen et Jahoda, 1986) et de porter « son attention sur ce qu'il se passe quand rencontre [ou comparaison] il y a » (Gajardo, Dervin et Lavanchy, 2011, p. 8), quel est ou quels sont le(s) point(s) de convergence(s) entre les différents travaux se réclamant d'une approche interculturelle? Peut-on identifier une conception partagée - ou paradigmatique - du terme lorsqu'il est mobilisé dans le champ scientifique? Peut-on dégager des épistémologies, des méthodologies, ou pour le moins des pratiques et des caractéristiques scientifiques communes à ce champ de recherche? Bref, sans craindre les abîmes sémantiques : quelle est la « culture » scientifique de la recherche interculturelle?

Reflétant les débats et les hésitations des sociétés, parfois en les devançant - on ne peut que constater qu'on trouve là aussi une diversité de perspectives. Pour certains, I' « interculturel » dans le champ scientifique définit une approche particulière, à la croisée des disciplines; pour d'autres, c'est un incontournable, voire un objet en soi. Dans ce cas de figure, d'adjectif, le terme devient substantif. Pour d'autres encore, c'est une idéologie que l'on nomme interculturalisme. Elle n'est alors certainement pas un objet de science, il faut plutôt en démonter les rouages pour ne pas y succomber. Enfin, la relation entre les cultures que le terme interculturel implique est comprise comme une relation théorique et pas nécessairement inscrite dans la réalité. On voit ainsi des études comparatives (sur le développement de l'enfant par exemple) qui se considèrent comme interculturelles parce qu'elles comparent les cultures entre elles. "Inter» n'est plus ici le résultat d'un contact, ce qui surgit de l'interaction entre altérités, mais une opération intellectuelle permettant de mettre ensemble ce qui ne l'aurait pas été dans la réalité et d’observer ce qui se trouve dans les interstices de cet assemblage artificiel.

Dans le cadre de ce premier numéro d'Alterstice, il nous est apparu indispensable de soulever ces questions. En effet, "faire de la recherche interculturelle » implique aussi de la penser, de s'interroger sur les conceptions, les usages, les épistémologies et les méthodologies que nous, chercheures et chercheurs, associons à ce terme qui qualifie nos pratiques scientifiques ou nos objets de recherche. Ces interrogations sont une invitation que nous formulons à l'égard de la revue, au-delà de ce premier numéro, et à tous ceux et celles qui s'identifient d'une manière ou d'une autre à ce champ de recherche: contribuons au débat, osons la critique, faisons preuve d'innovation et de créativité, mais surtout soyons rigoureux scientifiquement et intellectuellement dans les usages que nous faisons de cette notion et de ses dérivés (culture, multi-, trans-, etc.). C'est la condition sine qua non pour que la recherche interculturelle ne se réduise pas à un fourre-tout dont le seul point commun serait d'être à l'interstice de quelque chose. Tout comme il y a débat sur la notion de culture depuis plusieurs décennies, le débat sur les sens du terme interculturel va se poursuivre. Certains voudront voir sa disparition, d'autres sa reconnaissance comme discipline à part entière. Probablement que la diversité des acceptions - et les échanges à ce propos - sont la meilleure façon de faire vivre la recherche sur des objets complexes comme ceux que la recherche interculturelle tente de saisir. 


\section{Le défi des diversités}

Le paradoxe et l'un des défis majeurs de la recherche interculturelle est qu'au cœur de la définition, de l'objet et de l'identité de ce domaine d'étude en quête d'unité épistémologique et méthodologique se trouve précisément la notion de diversité : diversité culturelle, définie comme objet d'étude privilégié (mais qu'en est-il des autres diversités?), diversité des disciplines et des approches méthodologiques qui y contribuent, diversité des conceptions de la culture et de l'interculturalité.

À cet égard, les articles réunis ici reflètent bien la diversité de perspectives de la recherche interculturelle mentionnée plus haut, et la difficulté que semble poser à chacun l'exercice de définir, sans la figer, sa conception de l'« interculturel » et d'interroger de front les usages de la notion dans les champs où elle est mobilisée. Ainsi, plutôt qu'une discussion sur le terme - comme nous l'invitions à le faire dans le cadre de l'appel à articles de ce premier numéro - les contributions retenues nous fournissent des indices permettant de comprendre les réserves et les freins qui s'expriment à l'égard de cette notion et, surtout, nous donnent un aperçu de la diversité des conceptions, usages et ancrages disciplinaires qu'elle offre.

Ainsi, pour Margalit Cohen-Emerique, l'interculturel (elle emploie le substantif) est à la fois un champ de recherche, une conception sociopolitique du "vivre ensemble ", une approche en formation et en intervention et l'interaction entre deux identités qui se donnent mutuellement sens. L'interculturel comme champ de recherche porterait sur trois objets différents : la diversité culturelle (ce qui inclut la comparaison d'un même phénomène d'une culture à l'autre), les changements lors du contact de cultures (acculturation) et les processus d'interaction entre individus et groupes de cultures différentes (discrimination et stéréotype, mais aussi pédagogie, communication ou management interculturel). En tant que conception sociopolitique relative à la gestion de la diversité culturelle au sein d'une même société, l'interculturel est comparé au multiculturalisme, à l'intégration, à l'assimilation et à la lutte contre la discrimination. Margalit Cohen-Emerique rappelle les trois perspectives épistémologiques proposées par Abdallah-Pretceille : subjectiviste, interactionniste et situationniste. Elle propose également une « approche interculturelle " pouvant guider à la fois les formations à l'intervention auprès de populations " autres " et les interventions elles-mêmes, que ce soit en service social ou dans d'autres cadres institutionnels. Cette approche implique de remettre sans cesse en question la situation et ses propres hypothèses pour construire une compréhension plus fine, tout en entrant dans un processus de négociation de sens. Pour conclure, l'auteur définit l'interculturel comme l'interaction de deux identités qui se donnent mutuellement un sens dans un contexte à définir à chaque fois. À la lumière de cet article, la notion d'interculturel est, pour Margalit Cohen-Emerique, polysémique. Elle qualifie aussi bien des contextes macrosociaux (comme des choix politiques d'États nations) que des contextes microsociaux (comme des interactions entre usagers migrants et agents institutionnels). Cet ensemble est traversé par deux constantes constitutives d'un interculturel qui transcenderait ces définitions : d'une part les ancrages socioculturels de chaque humain et d'autre part l'interaction et son encadrement, nécessaire pour qu'elle aboutisse à la négociation de sens.

D’entrée de jeu, Marie Mc Andrew postule qu'il existe un paradigme interculturel. Cependant, ce paradigme serait de peu d'utilité dans la problématique que l'auteure traite, à savoir la question du voile islamique à l'école. Ce paradigme, non explicité (mais Marie Mc Andrew cite plusieurs auteurs qui le décrivent et en discutent), rencontrerait d'un point de vue descriptif des obstacles spécifiques sur les questions religieuse et politique, qu'il est par ailleurs essentiel d'aborder pour saisir les enjeux autour du voile. D'un point de vue normatif, les croyances religieuses évoquées pour justifier le voile seraient difficilement compatibles avec la recherche de compromis social propre à ce paradigme. C'est pourquoi elle lui préfère le paradigme de l'ethnicité, qui comporte plusieurs conceptions et approches théoriques. Le texte décrit ces diverses conceptions et en discute, et met en évidence leur efficacité à éclairer la problématique du voile. Les conceptions univoques de l'ethnicité sont l'essentialisme culturel, le constructivisme hétérocentriste (les ethnicités se construisent sous l'effet de pression externe ou interne au groupe; elles seraient le résultat de relations de pouvoir) et le constructivisme individualiste (l'ethnicité est le résultat d'un choix individuel). Cette dernière serait la conception favorite des tenants du paradigme interculturel pour qui l'interaction et la négociation au sein d'institutions communes seraient l'objectif à atteindre. La conception multivoque établit l'ethnicité " comme triple produit de la frontière externe, de la frontière interne et de la médiation par le Sujet ». Malgré les limites du paradigme interculturel soulignées dans l'analyse préalable, 
l'auteure est d'avis qu'il regagne en légitimité avec cette conception de l'ethnicité. En effet, il s'agit dans ce cas non pas de valoriser une explication plutôt qu'une autre, mais bien d'analyser chaque phénomène selon trois perspectives: les relations entre groupes, les relations internes aux groupes et la capacité de chaque individu à faire des choix et à négocier. Mettre à jour la complexité du jeu entre ces trois dynamiques oblige à contextualiser tout phénomène et rend l'analyse plus descriptive que prédictive. Ce serait là sa limite majeure, outre le fait qu'elle est beaucoup moins sécurisante que les conceptions univoques.

Pour Margarita Sanchez-Mazas et Raquel Fernandez-Iglesias, il y aurait un "paradigme interculturel » dans le monde de l'éducation, paradigme imprégnant les politiques éducatives et la formation des enseignants. Ce paradigme aurait permis une prise de conscience de l'hétérogénéité des publics scolaires, mais aurait aussi tendance à réduire les explications de situations complexes à la seule dimension culturelle. Reconstituant historiquement l'émergence de ce paradigme, les auteures montrent comment le handicap socioculturel devient la nouvelle explication simpliste pour tous les maux de l'école. Leur argumentation s'appuie sur des travaux en sociologie de l'éducation, en anthropologie et surtout en psychologie sociale, dont elles soulignent l'importance. Cette discipline scientifique serait incontournable pour aider les enseignants à comprendre les dynamiques identitaires et les processus de construction de la différence culturelle. Elle permettrait de décloisonner l'interculturel pour l'articuler à d'autres enjeux tout aussi importants. Pourtant, les auteures ne définissent pas vraiment ce qu'est I' « interculturel » pour elles. Elles emploient aussi bien le substantif que l'adjectif, et en font usage en les rattachant à un paradigme et à un champ de recherche lui-même associé à des situations de mixité culturelle en contexte scolaire. La notion est circonscrite à l'étude des interactions entre porteurs de cultures différentes (dans le cadre scolaire).

Ancrée disciplinairement en anthropologie sociale, la contribution de Louis-Jacques Dorais nous invite à réfléchir à la réinvention d'une langue autochtone disparue depuis 150 ans, le wendat (Canada). Décrivant le processus de cette reconstitution, et les conditions historiques y présidant, cet auteur nous montre comment une communauté peut être amenée à - ou plutôt choisit sciemment de - recréer une différenciation entre elle et les autres communautés, en particulier le groupe dominant, et de ce fait établit un nouvel espace interculturel (qui n'est justement pas un espace de rencontre, mais bien de mise à distance) là où il ne semblait pas y en avoir. Ce serait, selon l'auteur, le résultat d'un processus sociopolitique ancien qui trouverait son actualisation aujourd'hui étant donné le niveau de satisfaction des besoins premiers de cette communauté. Étonnamment, le fait que la communauté ait atteint un très haut niveau de ressemblance (socioéconomique, phénotypique, éducatif...) avec le groupe dominant permettrait justement d'investir le champ symbolique de la langue et de créer cet espace interculturel. Pour Louis-Jacques Dorais, I'interculturel résulte donc d'un processus construit socialement, politiquement et historiquement. C'est un espace symbolique qui met une distance entre deux groupes, ce serait en quelque sorte l'épaisseur de la frontière ethnique de Barth (1969).

Enfin, Karoline Truchon commence son article en rappelant le flou qui existe autour de la notion de culture malgré des décennies de recherches anthropologiques. Ancrant sa réflexion dans la recherche sur le vécu de la pauvreté, elle montre d'abord comment une construction théorique se voulant ouverte sur la culture en la déclinant « en facteurs et dimensions " finit par exclure un autre concept, la structure, qui lui est complémentaire plus qu'opposé. Elle pose tout de même que cette déclinaison permet une ouverture vers trois paradigmes: le multi-, l'inter- et le trans-culturel, ce que le concept de culture employé de façon réifiée ne permet pas. Le multiculturel, apparenté à l'anthropologie "traditionnelle » qui incarcérerait les individus dans leurs différences culturelles, ne serait que la juxtaposition de ces différences sans réelle rencontre entre elles. L'interculturel serait lui le paradigme du contact, de l'interaction et de la construction d'une culture commune à partir des cultures en contact. Le troisième paradigme permettrait de dépasser les frontières de la culture pour créer des liens qui les transcenderaient. Karoline Truchon expose des observations de son terrain de thèse, effectué dans un HLM de Montréal. Elle montre ainsi, à l'aide du cadre d'analyse de Small, Harding et Lamont, comment la culture peut être une notion favorisant l'intervention auprès de jeunes vivant en milieu précaire. Cependant, cela est vrai seulement si on n'aborde pas la question de l'appartenance culturelle de front (par exemple en organisant des soupers " culturels »). II s'agit de la prendre au sérieux si elle surgit, mais pas de la mettre en avant artificiellement. Cette approche serait, selon l'auteure, de l'ordre du paradigme transculturel. Un paradigme qui serait inquiétant pour les chercheurs parce que

Alterstice - Revue Internationale de la Recherche Interculturelle, vol. 1, $n^{\circ} 1$ 
fluide, nécessitant des redéfinitions permanentes et probablement des analyses contextualisées et plus descriptives que prédictives (pour employer les mots de Mc Andrew).

Les articles thématiques de ce numéro se terminent par un entretien croisé entre deux chercheurs spécialistes des questions interculturelles en éducation, Tania Ogay, et en management, Jacques Proulx. Par l'échange de leurs points de vue épistémologiques, plutôt convergents, ils nous éclairent sur les usages et mésusages du terme, ainsi que sur les tendances en recherche interculturelle. Selon eux, le terme recouvre de nombreuses réalités, parfois contradictoires. II n'y a pas une discipline interculturelle, mais bien la nécessité d'une interdisciplinarité pour étudier des objets complexes. II y a également nécessité d'intersubjectivité, pour tendre vers l'objectivité qui n'est finalement jamais atteinte. Si ce n'est pas une discipline, ce peut être une thématique, mais il faudrait alors employer le terme interculturalité, or c'est rarement le cas. II reste alors la perspective - ou l'approche interculturelle qui transcende les disciplines pour prendre la culture au sérieux et développer la décentration. Cependant, les chercheurs attirent notre attention sur le fait que la décentration n'est pas le constructivisme total, une forme radicale de relativisme qui diluerait la notion de culture dans une perspective ultra-individualiste et purement interactionniste. La décentration est cette capacité à surpasser son égocentrisme de chercheur (ou d'intervenant) et son ethnocentrisme très humain pour saisir son objet de recherche en contexte et en nuances. Ogay et Proulx soulignent le fait que la recherche interculturelle ne se fait pas sans tensions. Vivre et penser la diversité en même temps est un véritable défi. Or les intervenants, quels que soient leurs domaines (éducation, santé, management...), sont dans l'action. Ils vivent la diversité dans l'immédiat et n'ont que peu de temps pour y penser. Au contraire des décideurs, qui y pensent sans vraiment sortir de leurs réseaux sociaux habituels, parfois loin de la diversité. Certains chercheurs occupent la place intermédiaire, ils sont dans la posture de pouvoir observer la vie quotidienne et prendre le temps d'y penser. Ainsi la recherche interculturelle peut avoir des retombées concrètes dans la communauté, mais le chercheur ne doit pas confondre faits et valeurs, et se transformer en missionnaire de la cause interculturelle. Un biais que les deux interlocuteurs disent rencontrer fréquemment et qui nuit à l'image de la recherche interculturelle.

\section{Références bibliographiques}

Barth, F. (1969). Ethnic groups and boundaries : the social organization of culture difference. London : Allen \& Unwin.

Conseil de l'Europe (2008). Livre blanc sur le dialogue interculturel du Conseil de l'Europe. Strasbourg : Conseil de l'Europe.

Dasen, P. et Jahoda, G. (1986). Preface. Cross-cultural human development [Special Issue]. International journal of behavioral development, 9(4), 413-416.

Gajardo, A., Dervin, F. et Lavanchy, A. (2011). « United Colors Of... Interculturel » ? Usages, pièges et perspectives d'un terme plurivoque. Dans A. Lavanchy, A. Gajardo et F. Dervin (dir.), Anthropologie de l'interculturalité (p.7-43). Paris : L'Harmattan. 